\title{
New Interpretation of Newton's Law of Universal Gravitation
}

\section{Dalgerti L. Milanese}

Electrical Engineering Department, State University Júlio de Mesquita Filho-UNESP, Ilha Solteira, Brazil Email: dalgerti@hotmail.com

How to cite this paper: Milanese, D.L. (2017) New Interpretation of Newton's Law of Universal Gravitation. Journal of High Energy Physics, Gravitation and Cosmology, 3, 600-623.

https://doi.org/10.4236/jhepgc.2017.34046

Received: June 5, 2017

Accepted: September 22, 2017

Published: September 26, 2017

Copyright $\odot 2017$ by authors and Scientific Research Publishing Inc. This work is licensed under the Creative Commons Attribution International License (CC BY 4.0).

http://creativecommons.org/licenses/by/4.0/

(c) (i) Open Access

\begin{abstract}
Elliptical motions of orbital bodies are treated here using Fourier series, Fortescue sequence components and Clarke's instantaneous space vectors, quantities largely employed on electrical power systems analyses. Using this methodology, which evidences the analogy between orbital systems and autonomous second-order electrical systems, a new theory is presented in this article, in which it is demonstrated that Newton's gravitational fields can also be treated as a composition of Hook's elastic type fields, using the superposition principle. In fact, there is an identity between the equations of both laws. Furthermore, an energy analysis is conducted, and new concepts of power are introduced, which can help a better understanding of the physical mechanism of these quantities on both mechanical and electrical systems. The author believes that, as a practical consequence, elastic type gravitational fields can be artificially produced with modern engineering technologies, leading to possible satellites navigation techniques, with less dependency of external sources of energy and, even, new forms of energy sources for general purposes. This reinterpretation of orbital mechanics may also be complementary to conventional study, with implications for other theories such as relativistic, quantum, string theory and others.
\end{abstract}

\section{Keywords}

Orbital Motions, Gravity, Newton's Second Law, Hook's Elastic Forces, Fourier, Electrical Circuits, Transformations of Fortescue and Clarke, Instantaneous Space Vectors, Instantaneous Complex Power

\section{Introduction}

On steady-state analyses of polyphase electrical circuits, operating under unbalanced and sinusoidal conditions, usually, electrical engineers use Fortescue's method [1] to decompose those circuits on sets of balanced circuits, called posi- 
tive, negative and the zero-sequence symmetrical circuits. The correspondent electrical quantities are called symmetrical components, respectively, positive, negative and zero sequence components, whose respective phasors are of equal amplitudes and symmetrically spaced from each other, on a complex plane; excepted the zero-sequence phasors which are coincident.

On dealing with unbalanced and non-sinusoidal circuits, Fourier analysis is applied to each phase of the polyphase systems and, then, sets of symmetrical sequence circuits are obtained for each harmonic order, using the same method above described.

More recently, for three-phase circuits, a transformation, called Clarke's transformation [2], transforms those circuits on equivalent two-phase circuits, which brings all the information of the original circuits, i.e., unbalanced or balanced and/or sinusoidal or non-sinusoidal electrical quantities, such as voltages, currents, fluxes and charges. Clarke's transformation leads to a unique rotating vector, the instantaneous space vector (ISV) [2] [3] [4] [5] [6], on a complex plane.

After applying Clarke's transformation, then Fourier analysis is performed, and the positive complex Fourier coefficients, related to positive harmonic orders, are separated from the negative ones, using rotating unity vectors that rotate anti-clockwise and clockwise, respectively.

This is an alternative way to obtain the Fortescue sequence components, since they are, in fact, the Fourier coefficients themselves.

Fortescue's method is performed on the frequency domain. Clarke's, in turn, is performed on the time domain. In this work, Fourier analysis is applied to the study of elliptical orbital motions, which are represented on a complex plane, as compositions of two related orthogonal motions. In this way, all the mechanical quantities are treated as ISVs, and Clarke's inverse transformation can be performed to obtain the equivalent mechanical three-phase systems (other inverse transformations to polyphase systems are possible). In doing so, the analogy between orbital mechanical systems and three-phase electrical systems is evidenced. More precisely speaking, the dynamical behavior of elliptical orbital motions is analogous to the behavior of autonomous, second order, loss-less polyphase electrical circuits, with only reactive elements, operating under unbalanced and non-sinusoidal conditions.

The figures generated for the mechanical variables, on the complex plane, are all Lissajous figures of elliptical type.

Fourier analyses for orbital studies are not new, however, here, emphases are given to the geometric and dynamic properties of such motions, using the above-mentioned transformations of Fortescue and Clarke. Furthermore, the author develops a careful analysis on how the stored energy is distributed among the harmonic motions and their flux between the positive and negative sequence inside each harmonic motion, and among motions of different harmonic orders. Those energy fluxes can be better studied on future works, when the intention is 
how to manipulate them, when studying the possibility of new economical energy storage and consumption.

As a major contribution of this work, it is demonstrated that Newton's universal law for gravitational fields, the inverse square law, can be studied using Hook's law for elastic objects.

The author believes that new horizons are opened to the study and control of orbital dynamics, with possible applications of electric power systems control techniques, with low-loss reactive electromechanical elements.

Finally, the used concepts can also be extended to electrostatic forces and to the astronomy realms, and beyond the Newtonian mechanics.

\section{Discrete Fourier Series of the Position, Velocity and Force Vectors of Elliptical Orbital Motions, in Terms of Sequence Components}

For orbital motions, Kepler's equations are used for calculating the polar coordinates of the position vector $\tilde{\rho}$ as a function of time since perihelion. The amplitude of the position vector is given by

$$
\rho=\frac{a\left(1-e^{2}\right)}{1+e \cos \vartheta}
$$

and its angle by

$$
\varphi_{\rho} \equiv \vartheta
$$

where $a$ is the semi-major axis and $e$ and $\vartheta$ are respectively the eccentricity of the ellipse and the true anomaly.

Our method here, after obtaining in this way the position vector $\tilde{\rho}$, which is a periodic function of time, starts with a Fourier analysis of the motion, according to the Fourier theorem. Thus, the position vector is expressed as a Fourier series given by (all the vector quantities are represented on a complex plane $\alpha-\beta$ )

$$
\tilde{\rho}=\sum_{h=-N}^{N} \dot{\rho}_{h} \mathrm{e}^{i h \omega t}=\sum_{h=-N}^{N} \tilde{\rho}_{h}
$$

For circular and elliptical trajectories, the real and imaginary parts of $\tilde{\rho}$ are sinusoidal and periodical non-sinusoidal oscillations, respectively. Now, Equation (3) is expressed in terms of zero, positive and negative sequence components, changing the summation limits [6]:

$$
\tilde{\rho}=\dot{\rho}_{0}+\sum_{h=1}^{N} \tilde{\rho}_{h}^{+}+\sum_{h=1}^{N} \tilde{\rho}_{h}^{-}=\dot{\rho}_{0}+\tilde{\rho}_{+}+\tilde{\rho}_{-}
$$

Thus, the following identities show the position vector in terms of Kepler's equation (left-hand side) and Fourier series (right-hand side):

$$
\begin{gathered}
\rho=\frac{a\left(1-e^{2}\right)}{1+e \cos \vartheta} \equiv \operatorname{mag}(\tilde{\rho}), \\
\varphi_{\rho} \equiv \operatorname{ang}(\tilde{\rho}),
\end{gathered}
$$


where

$$
\begin{gathered}
\tilde{\rho}_{h}^{+}=\rho_{h}^{+} \mathrm{e}^{i\left(\varphi_{\rho h}^{+}\right)}=\rho_{h}^{+} \mathrm{e}^{i\left(h \omega_{1} t+\varphi_{\rho h 0}^{+}\right)}=\dot{\rho}_{h}^{+} \mathrm{e}^{i\left(h \omega_{1} t\right)}, \\
\tilde{\rho}_{h}^{-}=\rho_{h}^{-} \mathrm{e}^{-i\left(\varphi_{\rho h}^{-}\right)}=\rho_{h}^{-} \mathrm{e}^{-i\left(h \omega_{1} t-\varphi_{\rho h 0}^{-}\right)}=\dot{\rho}_{h}^{-} \mathrm{e}^{-i\left(h \omega_{1} t\right)}
\end{gathered}
$$

and $\omega_{1}$ is the fundamental frequency in $\mathrm{rad} / \mathrm{s}$.

The vectors $\tilde{\rho}_{h}^{+}$and $\tilde{\rho}_{h}^{-}$are rotating vectors with constant magnitudes and constant angular velocities, i.e., they describe uniform circular trajectories, the first one rotates on the counterclockwise direction, with angular velocity $h \omega_{1}$, and the latter on the clockwise direction with angular velocity $-h \omega_{1}$. The Fourier coefficients $\dot{\rho}_{h}^{+}$and $\dot{\rho}_{h}^{-}$are respectively the positive and negative harmonic phasors.

The sum of these two vectors

$$
\tilde{\rho}_{h}=\tilde{\rho}_{h}^{+}+\tilde{\rho}_{h}^{-}
$$

is another rotating vector, but with varying magnitude and angular velocity, which describes an ellipse illustrated in Figure 1 (Appendix A).

Thus, Equation (4) can also be written as

$$
\tilde{\rho}=\dot{\rho}_{0}+\sum_{h=1}^{N}\left(\tilde{\rho}_{h}^{+}+\tilde{\rho}_{h}^{-}\right)=\dot{\rho}_{0}+\sum_{h=1}^{N} \tilde{\rho}_{h},
$$

which shows that an elliptical trajectory described by the position vector $\tilde{\rho}$ is a composition of elliptical harmonic trajectories.

In turn, the velocity vector can be obtained in terms of $\tilde{\rho}^{+}$and $\tilde{\rho}^{-}$(Appendix B) as

$$
\begin{aligned}
\tilde{V} & =\frac{\mathrm{d} \tilde{\rho}}{\mathrm{d} t}=\frac{\mathrm{d} \tilde{\rho}_{h}^{+}}{\mathrm{d} t}+\frac{\mathrm{d} \tilde{\rho}_{h}^{-}}{\mathrm{d} t} \\
& =\tilde{V}^{+}+\tilde{V}^{-}=\sum_{h=1}^{N} \frac{\mathrm{d} \tilde{\rho}_{h}^{+}}{\mathrm{d} t}+\sum_{h=1}^{N} \frac{\mathrm{d} \tilde{\rho}_{h}^{-}}{\mathrm{d} t} \\
& =\sum_{h=1}^{N} i h \omega_{1} \tilde{\rho}_{h}^{+}-\sum_{h=1}^{N} i h \omega_{1} \tilde{\rho}_{h}^{-}=\sum_{h=1}^{N} i k_{h} \tilde{\rho}_{h}^{+}-\sum_{h=1}^{N} i k_{h} \tilde{\rho}_{h}^{-},
\end{aligned}
$$

where $k_{h}=h \omega_{1}$.

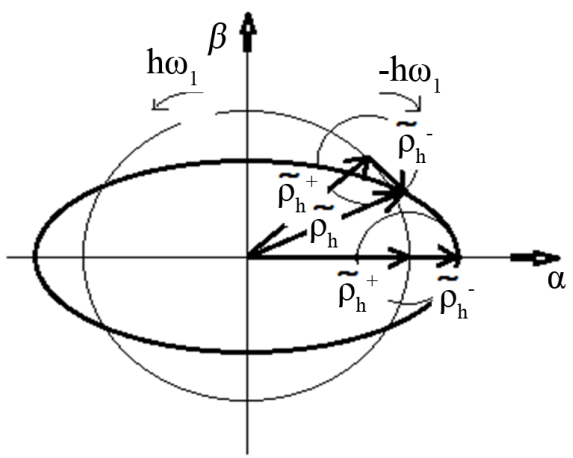

Figure 1. Illustration of the position vector trajectory for harmonic order $\boldsymbol{h}$, showing the positive and negative sequence motions, for the case of zero value for their initial angles. 
The velocity vector is usually obtained as the time derivative of the position vector from Clarke's equations, but its magnitude can be expressed by the Vis-viva equation; now it can also be obtained as a summation of terms with the harmonic position vectors $\tilde{\rho}_{h}^{+}$and $\tilde{\rho}_{h}^{-}$. Thus, its magnitude and angle can be given by the following identities:

$$
\begin{gathered}
V=\sqrt{G M\left(\frac{2}{\rho}-\frac{1}{a}\right)} \equiv \operatorname{mag}(\tilde{V}), \\
\varphi_{V} \equiv \operatorname{ang}(\tilde{V}) .
\end{gathered}
$$

Note that $\varphi_{V h}=\varphi_{\rho h}+\frac{\pi}{2}$, and that this orthogonality is related to the harmonic reactive power concept presented in chapter III. The total velocity $\tilde{V}$ is not orthogonal to the total position vector. Such properties from elementary kinematics are studied in detail in the same chapter, concerning the powers involved, and new power concepts are then presented.

And the gravitational force, in turn, is obtained from Newton's universal law equation, and now, alternatively, in terms of the harmonic position vectors $\tilde{\rho}_{h}^{+}$ and $\tilde{\rho}_{h}^{-}$

$$
\begin{aligned}
\tilde{F} & =m \frac{\mathrm{d} \tilde{V}}{\mathrm{~d} t}=m\left(\frac{\mathrm{d} \tilde{V}^{+}}{\mathrm{d} t}+\frac{\mathrm{d} \tilde{V}^{-}}{\mathrm{d} t}\right) \\
& =m\left(\sum_{h=1}^{N}-h^{2} \omega_{1}^{2} \tilde{\rho}_{h}^{+}+\sum_{h=1}^{N}-h^{2} \omega_{1}^{2} \tilde{\rho}_{h}^{-}\right) \\
& =-m\left(\sum_{h=1}^{N} k_{h}^{2} \tilde{\rho}_{h}^{+}+\sum_{h=1}^{N} k_{h}^{2} \tilde{\rho}_{h}^{-}\right)
\end{aligned}
$$

This conclusion stablishes that, on orbital motions, Newton's universal gravitational law, also known as Newton's inverse-square law, and Hook's elastic law can now be unified and written as an identity:

$$
\tilde{F}_{G}=-\frac{G M m}{\rho^{2}} \mathrm{e}^{i \varphi_{\rho}} \equiv-m\left(\sum_{h=1}^{N} k_{h}^{2} \tilde{\rho}_{h}^{+}+\sum_{h=1}^{N} k_{h}^{2} \tilde{\rho}_{h}^{-}\right) .
$$

Hook's law is evidenced on the right-hand sides of these identities, because each harmonic force component is proportional to the magnitude of the same harmonic order position vector, where Hook's elastic constant is $k_{h}^{\prime}=m k_{h}^{2}$.

Thus, gravitational forces can be given as a weighted sum of harmonic positions vectors. This identity can also be interpreted as a statement that the gravitational force is a summation of harmonic centripetal forces.

To obtain the harmonic phasors $\dot{\rho}_{h}^{+}$, the ISV $\tilde{\rho}$ is rewriting on new complex $\mathrm{d}-\mathrm{q}$ coordinate systems determined by the orthogonal unity vectors $u_{d h}=\mathrm{e}^{i h \omega_{1} t}$ and $u_{q h}=\mathrm{e}^{i h \omega_{1} t+\frac{\pi}{2}}$, which rotates on counterclockwise direction with angular velocity $h \omega_{1}$, where $h \omega_{1} t$ is measured from the real axis $\alpha$ from the $\alpha-\beta$ stationary coordinate system and, for $\dot{\rho}_{h}^{-}, \mathrm{d}$-q rotates on clockwise direction. 
On these new coordinate systems, each harmonic rotating vectors of order $h$, $\tilde{\rho}_{h}^{+}$and $\tilde{\rho}_{h}^{-}$, become the stationary vectors $\dot{\rho}_{h}^{+}$and $\dot{\rho}_{h}^{-}$, usually called phasors, and all the other rotating vectors of different orders have zero mean values of its real and imaginary parts. The fundamental angular velocity $\omega_{1}$ (fundamental frequency) is obtained from the third Kepplers' law, which gives the orbit period D.

This method is general for any closed elliptical orbit, considering that for each orbit, which are determined by the amplitude of its semi-major axis, its eccentricity, the mass of the central and the orbiting bodies, the gravitational constant and the period, the fundamental frequency in $\mathrm{Hz}$ is calculated as mentioned above, and the time step is chosen such as to avoid aliasing.

\section{Energy and Power Analysis}

An ideal orbital motion is an autonomous conservative (non-dissipative) second order system, i.e., a system with no external exchange of energy, with two internal storage energy elements: the gravitational field and the moving mass.

Considering this motion as a composition of two orthogonal motions, the kinetic and potential energies are exchanged between these two motions, in this way (Appendices $\mathrm{C}$ and $\mathrm{D}$ ):

For ideal circular motions, there is an exchange of kinetic energy between the two orthogonal motions, as well as of potential energy, but not between these two forms of energy, such that the sum of the kinetic energy and the sum of the potential energy are constant. That oscillating energies are related to force and velocity in quadrature and their rate of oscillation is called here imaginary power.

For ideal elliptical motions, in turn, there is also an exchange of kinetic energy between the two motions, as well as of potential energy, and an exchange of kinetic energy from one motion with that potential energy from the other motion, such that the sum of the kinetic energy and the sum of the potential energy are not constant. That oscillation of energy between the two forms is related to force and velocity in the same direction (but not in phase) and its rate of oscillation is called here real power.

The amount of this latter oscillating energy depends on the trajectory eccentricity.

There is no a consensual interpretation for the analogous forms of those energies on electrical circuits analyses [7].

\subsection{Kinetic Energy}

Considering the harmonic elliptical motion of order $h$, the correspondent harmonic kinetic energy, in terms of sequence components, is

$$
K_{h}=\frac{1}{2} m V_{h}^{2}=\frac{1}{2} m \tilde{V}_{h} \tilde{V}_{h}^{*}=\frac{1}{2} m\left[\left(V_{h}^{+}\right)^{2}+\left(V_{h}^{-}\right)^{2}+2 V_{h}^{+} V_{h}^{-} \cos \left(\varphi_{V h}^{+}-\varphi_{V h}^{-}\right)\right]
$$

The average value of this energy can be given as 


$$
K_{\text {havg }}=K_{h}^{+-}=\overline{\frac{1}{2} m V_{h}^{2}}
$$

where

$$
\begin{gathered}
K_{h}^{+-}=K_{h}^{+}+K_{h}^{-}, \\
K_{h}^{+}=\frac{1}{2} m\left(V_{h}^{+}\right)^{2}=\frac{Q_{h}^{+}}{2 h \omega_{1}}, \\
K_{h}^{-}=\frac{1}{2} m\left(V_{h}^{-}\right)^{2}=-\frac{Q_{h}^{-}}{2 h \omega_{1}},
\end{gathered}
$$

and $Q_{h}^{+}, Q_{h}^{-}$are respectively the positive and negative sequence harmonic imaginary (reactive) powers.

Now, in terms of sequence components, the total kinetic energy of the motion is given as

$$
\begin{aligned}
K & =\frac{1}{2} m V^{2}=\frac{1}{2} m \tilde{V} \tilde{V}^{*}=\frac{1}{2} m\left(\tilde{V}^{+}+\tilde{V}^{-}\right)\left(\tilde{V}^{+}+\tilde{V}^{-}\right)^{*} \\
& =\frac{1}{2} m\left[\left(V^{+}\right)^{2}+\left(V^{-}\right)^{2}+\tilde{V}^{+} \tilde{V}^{-^{*}}+\tilde{V}^{-} \tilde{V}^{+}\right] \\
& =\frac{1}{2} m\left[\left(V^{+}\right)^{2}+\left(V^{-}\right)^{2}+2 V^{+} V^{-} \cos \left(\varphi_{V+}-\varphi_{V-}\right)\right],
\end{aligned}
$$

where $\tilde{V}^{+}=V^{+} \mathrm{e}^{i \varphi_{V_{+}}}$and $\tilde{V}^{-}=V^{-} \mathrm{e}^{i \varphi_{V_{-}}}$, wherein both magnitudes and both angles vary at variable rate.

The average kinetic energy of the total motion during one cycle, can be given as

$$
K_{a v}=\sum_{h=1}^{N}\left(K_{h}^{+}+K_{h}^{-}\right) .
$$

The rate of the total kinetic energy variation is the total power exchanged between the gravitational field and the moving mass.

So far, we have seen that the elliptical motion of a satellite around a central body is composed of a summation of harmonic elliptical motions, each one concerned to the respective harmonic position vector. For every harmonic motion, there are potential and kinetic energies. Part of these energies is constant and another part oscillates between these two forms of energy. The amount of oscillating energies depends on the trajectory eccentricity. For example, a circular trajectory $(\mathrm{e}=0)$ has only constant energy stored, i.e., there is no energy exchanged between the potential field and the moving mass.

\subsection{Potential Energy}

Considering the gravitational potential energy referenced to a point at infinity,

$$
U_{G}=-F_{G} \rho
$$

and the potential elastic energy, referenced to the origin of the coordinate system,

$$
U_{\text {Hook }}=\frac{1}{2} F_{G} \rho \text {, }
$$


the gravitational energy, which is the sum of the kinetic and potential gravitational energies,

$$
E_{G}=K+U_{G},
$$

where $E_{G}<0$ and $U_{G}<0$; and the elastic energy, which is the sum of the kinetic and potential elastic energies,

$$
E_{\text {Hook }}=K+U_{\text {Hook }} \text {, }
$$

where $E_{\text {Hook }}>0$ and $U_{\text {Hook }}>0$, are related in the following way:

$$
E_{G}=-\frac{1}{2} E_{\text {Hook }},
$$

where

$$
E_{\text {Hook }}=\sum_{h=1}^{N} E_{\text {Hookh }}
$$

and

$$
\begin{gathered}
E_{\text {Hookh }}=K_{h}+U_{\text {Hook }}, \\
E_{\text {Hookh }}=\frac{1}{2} F_{h} \rho_{h} .
\end{gathered}
$$

And, from (13), we have

$$
F_{h}=-m k_{h}^{2} \rho_{h}
$$

\subsection{Power}

To deal with gravitational energies and their rate of variation in terms of Hook's equations, it is adopted in this work the instantaneous complex power (ICP) approach [3] [4] [5] [6] [8] [9]. This approach helps understanding how each harmonic motion contributes with the total power (see Appendix D).

For each harmonic pair of force and velocity, the ICP is

$$
\begin{aligned}
\tilde{S}_{h} & =\tilde{F}_{h} \tilde{V}_{h}^{*}=\left(\tilde{F}_{h}^{+}+\tilde{F}_{h}^{-}\right)\left(\tilde{V}_{h}^{+}+\tilde{V}_{h}^{-}\right)^{*} \\
& =\dot{F}_{h}^{+}\left(\dot{V}_{h}^{+}\right)^{*}+\dot{F}_{h}^{-}\left(\dot{V}_{h}^{-}\right)^{*}+\tilde{F}_{h}^{+}\left(\tilde{V}_{h}^{-}\right)^{*}+\tilde{F}_{h}^{-}\left(\tilde{V}_{h}^{+}\right)^{*} \\
& =S_{h}^{+}+S_{h}^{-}+\tilde{S}_{h}^{+-}+\tilde{S}_{h}^{-+} .
\end{aligned}
$$

The real part of $S_{h}^{+}$and $S_{h}^{-}, P_{h}^{+}$and $P_{h}^{-}$, respectively, are the positive and the negative sequence real powers and are nil in the case of non-dissipative motions (for example: no atmosphere resistance), and the imaginary part, $Q_{h}^{+}$ and $Q_{h}^{-}$, respectively, are the positive and the negative sequence imaginary powers and they are constant.

The remaining terms of Equation (30) are related to the power oscillation between the kinetic form and the gravitational form for each harmonic order motion. Thus, the subtotal power oscillation due to all harmonic pairs is (Appendix D1)

$$
\tilde{Q}_{\sum P h}=\tilde{Q}_{\Sigma P h}^{+-}+\tilde{Q}_{\Sigma P h}^{-+}=\sum \operatorname{Real}\left[\tilde{F}_{h}^{+}\left(\tilde{V}_{h}^{-}\right)^{*}+\tilde{F}_{h}^{-}\left(\tilde{V}_{h}^{+}\right)^{*}\right]
$$


There are also oscillating powers between the potential field and the moving mass due to forces and velocities of different harmonic orders (Appendix D2).

$$
\begin{aligned}
\tilde{Q}_{\sum P i j} & =\tilde{Q}_{\sum P i j}^{ \pm}+\tilde{Q}_{\sum P i j}^{\mp} \\
& =\sum_{i=1}^{N} \sum_{\substack{j=1 \\
j \neq i}}^{N} \operatorname{Real}\left[\tilde{F}_{i}^{+}\left(\tilde{V}_{j}^{+}\right)^{*}+\tilde{F}_{i}^{+}\left(\tilde{V}_{j}^{-}\right)^{*}+\tilde{F}_{i}^{-}\left(\tilde{V}_{j}^{+}\right)^{*}+\tilde{F}_{i}^{-}\left(\tilde{V}_{j}^{-}\right)^{*}\right]
\end{aligned}
$$

The total power oscillation between the gravitational potential field and the moving mass, which is the time derivative of the total kinetic energy is given by

$$
\tilde{Q}_{\sum P}=\tilde{Q}_{\sum P h}+\tilde{Q}_{\sum P i j}=\tilde{Q}_{\sum P h}^{ \pm}+\tilde{Q}_{\sum P h}^{\mp}+\tilde{Q}_{\sum P i j}^{ \pm}+\tilde{Q}_{\sum P i j}^{\mp} .
$$

\section{Case Study}

In this chapter, there are shown the discrete complex Fourier (DCF) analysis and synthesis results concerned to one cycle of variation of the position, velocity and force vectors. All the numerical operations were algebraic, developed in time domain.

Initially, the original position, velocity and force vectors are computed using the conventional laws for orbital motions, i.e., Kepler's equations, the Vis-viva equation and Newton's law for universal gravitation, respectively, except the velocity vector angle, not obtained from Vis-viva equation. The used approximation for obtaining the position vector is that presented in [10].

Then, for validating the proposed theory in this work, a Fourier analysis is performed on the original position vector, then it is synthetized, as well as, the velocity and force vectors, using the right-hand sides of the respective identities (5), (6), (10), (11) and (12), for one cycle of the orbit, and compared to those on the left hand sides.

All the results were computed using Google's Drive spreadsheets. Those obtained from the conventional methods will be referred to as Kepler's results, or original results. Those obtained by Fourier analysis and synthesis will be referred to as Hook's results, or computed results.

The orbital motion studied is a satellite's low earth orbit motion, with the following parameters: mass $m=1 \mathrm{~kg}$, the semi-major axis $a=8000000 \mathrm{~m}$, the initial instant $t_{0}=0 \mathrm{~s}$, the initial eccentric anomaly $E_{0}=10^{\circ}$, the gravitational constant multiplied by the earth mass $G M=3.986005 \mathrm{~m}^{3} / \mathrm{s}^{2}$ the calculated period is $P=7121.08 \mathrm{~s}$, and the fundamental frequency is $\omega_{1}=8.82 \times 10^{-4} \mathrm{rad} / \mathrm{s}$.

The calculated total energy (gravitational energy) using Vis-viva equation is $E_{G}=24912531.25 \mathrm{~J}$; the calculated average kinetic energy using equation (20) is $K_{a v}=24912.44 \mathrm{~kJ} \quad($ error $\%=0.00)$ and the position vector is sampled at constant rate with time step $84.77 \mathrm{~s}$, with a total of 84 samples/cycle.

The following results, computed for $e=0.3$ (only for analytical purpose, because for this eccentricity the satellite falls on the earth), show how the position vector at Perihelion and Aphelion is composed by the terms of equation (4). Referring to this equation, it was verified that all the harmonic position vectors line up at Perihelion and every even harmonic position vector subtracts at the Aphe- 
lion, shifting, in this way, the center of the ellipse from the center of the coordinate system. The total shifting is due to this one and to the shifting due to the zero-sequence vector in opposite direction, i.e., the Aphelion direction.

Starting with the focus distance $c=2400.00 \mathrm{~km}$ from $c=a e$, the position vector magnitude at Perihelion is $\rho_{\varphi_{\rho}=0}=a-c=5600.00 \mathrm{~km}$, and position vector magnitude at Aphelion is $\rho_{\varphi_{\rho}=180}=a+c=10400.00 \mathrm{~km}$.

The computed Hook's position vector magnitude at Perihelion $\rho_{\varphi_{\rho}=0}=5603.31 \mathrm{~km} \quad$ (error $\%=0.06$ ); and the computed Hook's focus distance $c=a-\rho_{\varphi_{\rho}=0}=8000.00-5603.31=2396.69 \mathrm{~km} \quad($ error $\%=-0.14)$.

The position vector magnitude at Perihelion of the computed ellipse, excluding the zero-sequence position vector $\dot{\rho}_{0}$ is

$$
\left|\left(\tilde{\rho}-\dot{\rho}_{0}\right)_{\varphi_{\rho}=0}\right|=\left|\left(\sum_{h=1}^{N} \tilde{\rho}_{h}\right)_{\varphi_{\rho}=0}\right|=9203.29 \mathrm{~km}
$$

where the zero-sequence position vector was computed as $\dot{\rho}_{0}=\rho_{0} \mathrm{e}^{i\left(\varphi_{\rho 0}\right)}=3600.00 \mathrm{e}^{i(180)} \mathrm{km}$. And the computed position vector magnitude at Aphelion, in the same way, is $\left|\left(\tilde{\rho}-\dot{\rho}_{0}\right)_{\varphi_{\rho}=180}\right|=\left|\left(\sum_{h=1}^{N} \tilde{\rho}_{h}\right)_{\varphi_{\rho}=180}\right|=10397.73 \mathrm{~km}$ (error \% $=-0.02)$.

Thus, by other way, the position vector magnitude at Perihelion of the computed ellipse including the zero sequence vector is

$$
\rho_{\varphi_{\rho}=0}=\left|\left(\sum_{h=1}^{N} \tilde{\rho}_{h}\right)_{\varphi_{\rho}=0}\right|+\rho_{0}=9203.29-3600.00=5603.29 \mathrm{~km}
$$

So, as we have mentioned above, these results showed how the position vector at Perihelion and Aphelion is composed by the terms of Equation (4).

Table 1 shows the computed complex Fourier sequences components (Fourier coefficients) of Hook's position vector for three eccentricity values (illustrated on Figure 3 and Figure 4). Note that the angles for each sequence component harmonic are equals, and all the angles are multiples of that one of fundamental frequency. In other words: The initial harmonic phase angles for the displacement vectors are multiple of the mean anomaly $M: \varphi_{\rho h 0}^{+}=\varphi_{\rho h 0}^{-}=h M$

Table 2 shows the results for the angular momentum calculation, for three orbit positions and $e=0.3$. These results show the precision of the method on the computation of the position and velocity vectors, which can also be seen in the figures ahead.

Table 3 was obtained for three orbit positions and three eccentricity values.

The following computed figures are referred to as "original" for Keplers' results and "computed" for Hook's results. They illustrate the principal characteristics of the studied orbital motion and show the numerical precision of the results, for eccentricity $e=0.3$.

Figure 2 shows the satellite's trajectory, i.e. the position vector variation; Figure 3 and Figure 4 show the harmonic vector spectrum (obtained from the results of Table 1). 
Table 1. Computed complex fourier sequences components (fourier coefficients) of Hook's position vector for three eccentricity values.

\begin{tabular}{|c|c|c|c|c|c|c|}
\hline \multirow{2}{*}{$\mathrm{h}$} & \multicolumn{6}{|c|}{ Position vector-Amplitude (m), Angle (degrees) } \\
\hline & \multicolumn{3}{|c|}{ Positive sequence } & \multicolumn{3}{|c|}{ Negative sequence } \\
\hline 1 & $7.96 \mathrm{E}+06 / 9.01$ & $7.84 \mathrm{E}+06 / 8.01$ & $7.64 \mathrm{E}+06 / 7.02$ & $1.00 \mathrm{E}+04 / 9.01$ & $4.05 \mathrm{E}+04 / 8.01$ & $9.29 \mathrm{E}+04 / 7.02$ \\
\hline 2 & $3.97 \mathrm{E}+05 / 18.01$ & $7.76 \mathrm{E}+05 / 16.02$ & $1.12 \mathrm{E}+06 / 14.03$ & $3.34 \mathrm{E}+02 / 18.01$ & $2.70 \mathrm{E}+03 / 16.02$ & $9.40 \mathrm{E}+03 / 14.03$ \\
\hline 3 & $2.97 \mathrm{E}+04 / 27.02$ & $1.15 \mathrm{E}+05 / 24.03$ & $2.46 \mathrm{E}+05 / 21.05$ & $1.88 \mathrm{E}+01 / 27.02$ & $3.03 \mathrm{E}+02 / 24.03$ & $1.58 \mathrm{E}+03 / 21.05$ \\
\hline 4 & $2.63 \mathrm{E}+03 / 36.02$ & $2.03 \mathrm{E}+04 / 32.04$ & $6.40 \mathrm{E}+04 / 28.06$ & $8.13 \mathrm{E}-01 / 36.02$ & $2.49 \mathrm{E}+01 / 32.04$ & $8.59 \mathrm{E}+02 / 28.06$ \\
\hline 5 & $2.56 \mathrm{E}+02 / 45.03$ & $3.91 \mathrm{E}+03 / 40.05$ & $1.80 \mathrm{E}+04 / 35.08$ & $8.91 \mathrm{E}-02 / 45.03$ & $1.83 \mathrm{E}+00 / 40.05$ & $5.80 \mathrm{E}+01 / 35.08$ \\
\hline 6 & $2.70 \mathrm{E}+01 / 54.03$ & $8.69 \mathrm{E}+02 / 48.06$ & $6.63 \mathrm{E}+03 / 42.09$ & $5.98 \mathrm{E}-01 / 54.03$ & $7.74 \mathrm{E}+01 / 48.06$ & $1.35 \mathrm{E}+03 / 42.09$ \\
\hline 8 & $2.56 \mathrm{E}-01 / 72.04$ & $3.06 \mathrm{E}+01 / 64.08$ & $4.65 \mathrm{E}+02 / 56.12$ & $7.21 \mathrm{E}-04 / 72.04$ & $3.35 \mathrm{E}-01 / 64.08$ & $1.08 \mathrm{E}+01 / 56.12$ \\
\hline 9 & $3.14 \mathrm{E}-02 / 81.05$ & $7.63 \mathrm{E}+00 / 72.09$ & $1.79 \mathrm{E}+02 / 63.14$ & $1.19 \mathrm{E}-04 / 81.05$ & $1.13 \mathrm{E}-01 / 72.09$ & $5.73 \mathrm{E}+00 / 63.14$ \\
\hline 10 & $3.17 \mathrm{E}-03 / 90.05$ & $1.55 \mathrm{E}+00 / 80.10$ & $5.47 \mathrm{E}+01 / 70.15$ & $1.49 \mathrm{E}-05 / 90.07$ & $2.82 \mathrm{E}-02 / 80.10$ & $2.09 \mathrm{E}+00 / 70.15$ \\
\hline 11 & $3.07 \mathrm{E}-04 / 99.06$ & $2.99 \mathrm{E}-01 / 88.11$ & $1.58 \mathrm{E}+01 / 77.17$ & $1.81 \mathrm{E}-06 / 99.26$ & $7.36 \mathrm{E}-03 / 88.11$ & $9.36 \mathrm{E}-01 / 77.17$ \\
\hline 12 & $2.89 \mathrm{E}-05 / 108.07$ & $5.63 \mathrm{E}-02 / 96.12$ & $4.41 \mathrm{E}+00 / 84.18$ & $2.18 \mathrm{E}-07 / 109.32$ & $1.85 \mathrm{E}-03 / 96.12$ & $3.78 \mathrm{E}-01 / 84.18$ \\
\hline 13 & -------- & $1.09 \mathrm{E}-02 / 104.13$ & $1.41 \mathrm{E}+00 / 91.20$ & --------- & $7.93 \mathrm{E}-05 / 104.14$ & $2.71 \mathrm{E}-02 / 91.20$ \\
\hline 14 & --------- & $2.06 \mathrm{E}-03 / 112.14$ & $4.51 \mathrm{E}-01 / 98.21$ & ---------- & $8.60 \mathrm{E}-06 / 112.14$ & $5.93 \mathrm{E}-03 / 98.21$ \\
\hline 15 & ------ & $2.65 \mathrm{E}-05 / 120.14$ & $1.00 \mathrm{E}-02 / 105.23$ & -------- & $3.17 \mathrm{E}-06 / 120.02$ & $1.49 \mathrm{E}-03 / 105.23$ \\
\hline
\end{tabular}

Table 2. Computed Hook's angular momentum $(e=0.3)$

\begin{tabular}{cc}
\hline True anomaly $(\mathrm{rad})$ & Angular Momentum $\left(\mathrm{m}^{2} \mathrm{~kg} / \mathrm{s}\right)$ \\
\hline 0.24 & $53,889,267,155.99$ \\
0.38 & $53,881,103,300.35$ \\
0.52 & $53,877,893,316.53$ \\
\hline
\end{tabular}

Table 3. Computed Hook's results for the position, velocity e force vector for three positions of the position vector and three eccentricity values.

\begin{tabular}{|c|c|c|c|c|c|c|c|c|}
\hline \multicolumn{3}{|c|}{ Position vector-Amplitude (m), Angle (degrees) } & \multicolumn{3}{|c|}{ Velocity vector-Amplitude (m/s), Angle (degrees) } & \multicolumn{3}{|c|}{$\begin{array}{l}\text { Force vector-Amplitude (N), Angle } \\
\text { (degrees) }\end{array}$} \\
\hline $\mathrm{e}=0.1$ & $\mathrm{e}=0.2$ & $\mathrm{e}=0.3$ & $\mathrm{e}=0.1$ & $\mathrm{e}=0.2$ & $\mathrm{e}=0.3$ & $\mathrm{e}=0.1$ & $\mathrm{e}=0.2$ & $\mathrm{e}=0.3$ \\
\hline Mo $=9.01$ & $\mathrm{Mo}=8.01$ & $\mathrm{Mo}=7.02$ & Мo $=9.01$ & Mo $=8.01$ & $\mathrm{Mo}=7.02$ & $\mathrm{Mo}=9.01$ & $\mathrm{Mo}=8.01$ & Mo = 7.02 \\
\hline $7.21 \mathrm{E}+06 / 11.08$ & $6.43 \mathrm{E}+06 / 12.35$ & $5.65 \mathrm{E}+06 / 13.9$ & $7.79 \mathrm{E}+03 / 100.02$ & $8.62 \mathrm{E}+03 / 100.11$ & $9.56 \mathrm{E}+06 / 100.27$ & $7.66 / 191.07$ & $9.66 / 192.3$ & $12.64 / 194.00$ \\
\hline $7.23 \mathrm{E}+06 / 116.31$ & $6.46 \mathrm{E}+06 / 18.82$ & $5.71 \mathrm{E}+06 / 22.02$ & $7.78 \mathrm{E}+03 / 104.79$ & $8.58 \mathrm{E}+03 / 105.54$ & $9.48 \mathrm{E}+03 / 106.64$ & $7.63 / 196.30$ & $9.57 / 198.81$ & $12.39 / 202.06$ \\
\hline $7.25 \mathrm{E}+06 / 21.52$ & $6.51 \mathrm{E}+06 / 25.21$ & $5.80 \mathrm{E}+06 / 29.93$ & $7.76 \mathrm{E}+03 / 109.55$ & $8.52 \mathrm{E}+03 / 110.93$ & $9.37 \mathrm{E}+03 / 112.90$ & $7.59 / 201.51$ & $9.44 / 205.19$ & $12.04 / 209.91$ \\
\hline
\end{tabular}

Figures 5-7 show, respectively, the magnitude variation of the position, the velocity and the force vectors, during one cycle, for the computed values from Kepler's equations (original), compared with those obtained from Hook's (computed). 


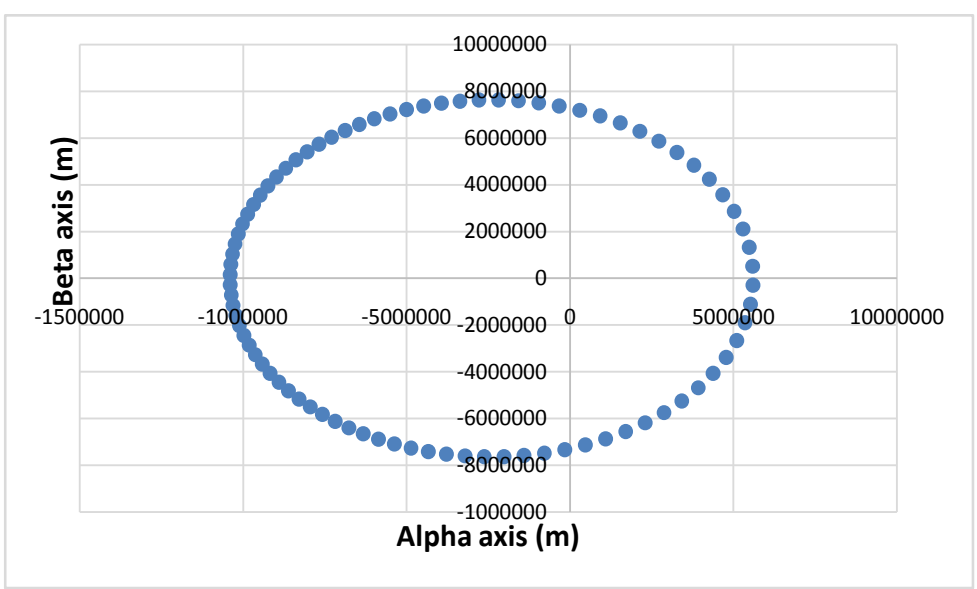

Figure 2. Keppler's trajectory.

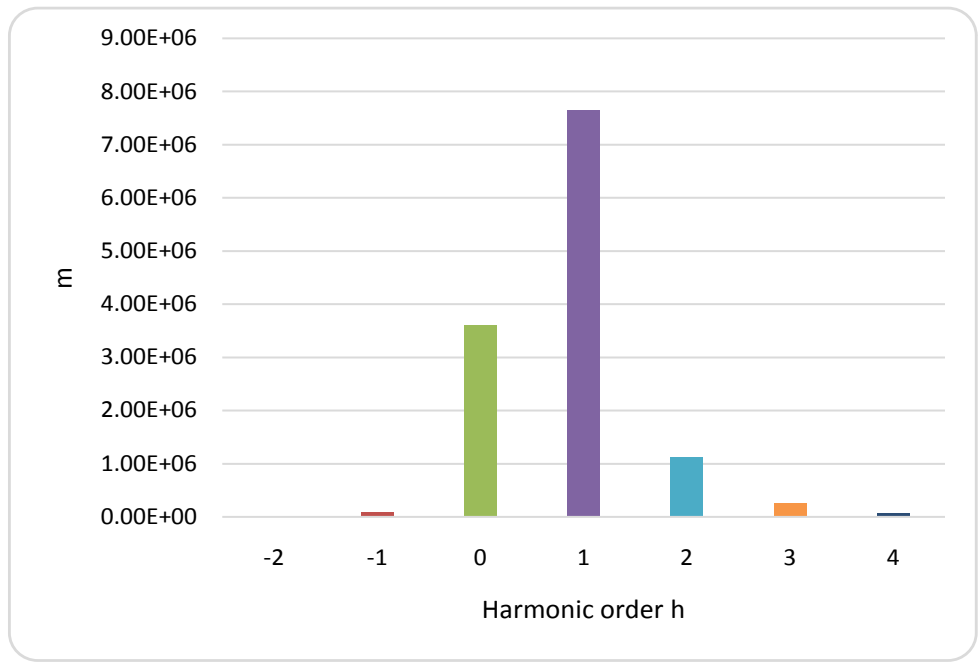

Figure 3. Position vector spectrum.

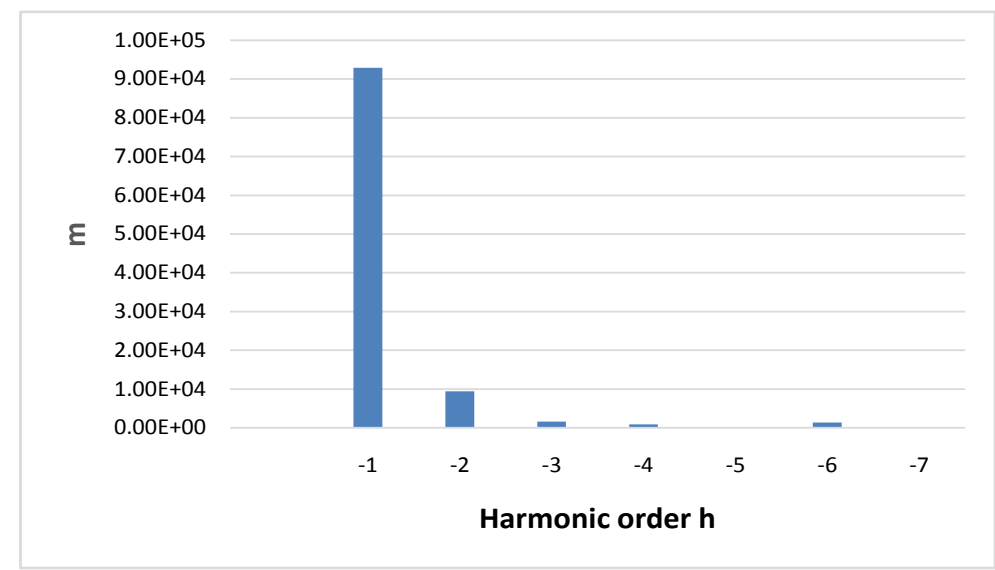

Figure 4. Position vector spectrum (negative sequence).

Figure 8 and Figure 9 show, respectively, the energy balance for the gravitational and elastic systems for instantaneous quantities computed at the initial 


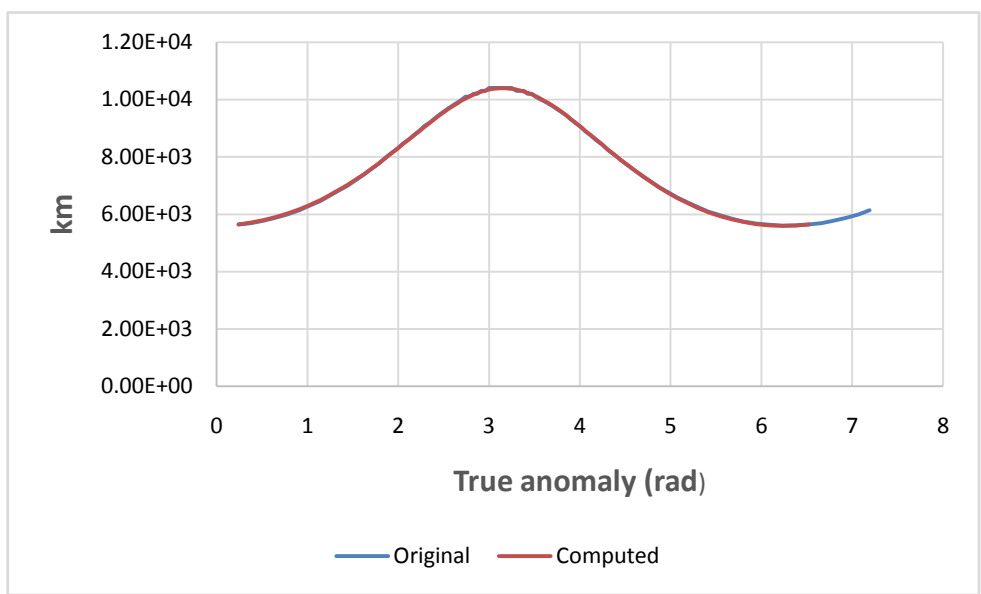

Figure 5. Position vector magnitude variation.

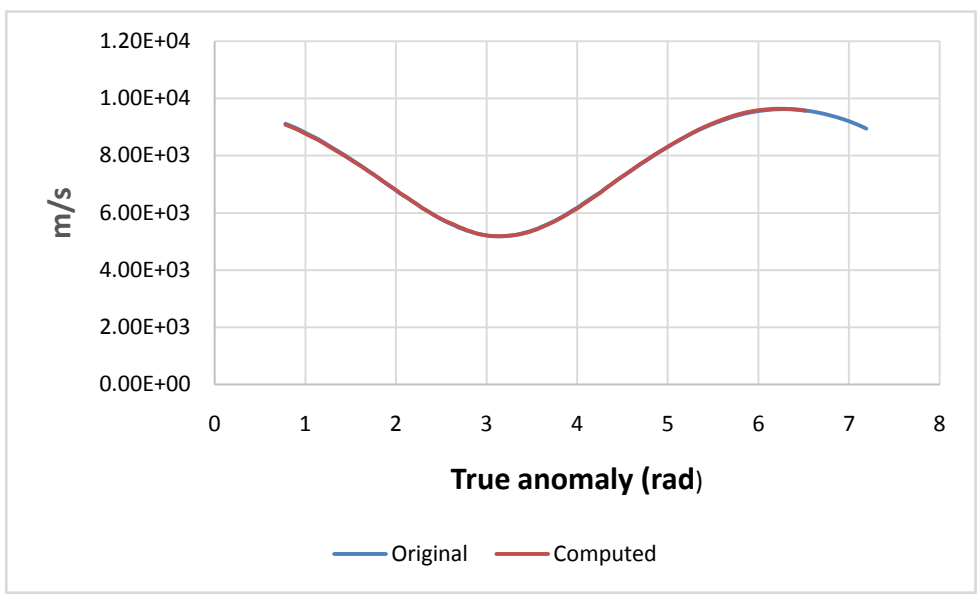

Figure 6. Velocity vector magnitude variation.

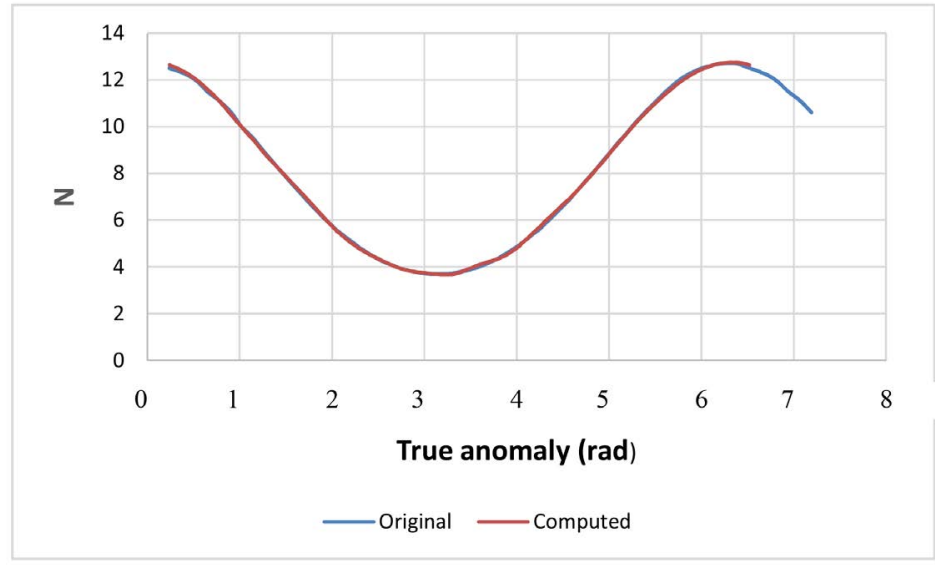

Figure 7. Force vector magnitude variation.

instant $t_{0}$, according the equations presented in Chapter III, where (a) kinetic, (b) average kinetic , (c) potential, $(d)$ average potential, $(a+c)$ total energy, $(b+$ d) average total. 


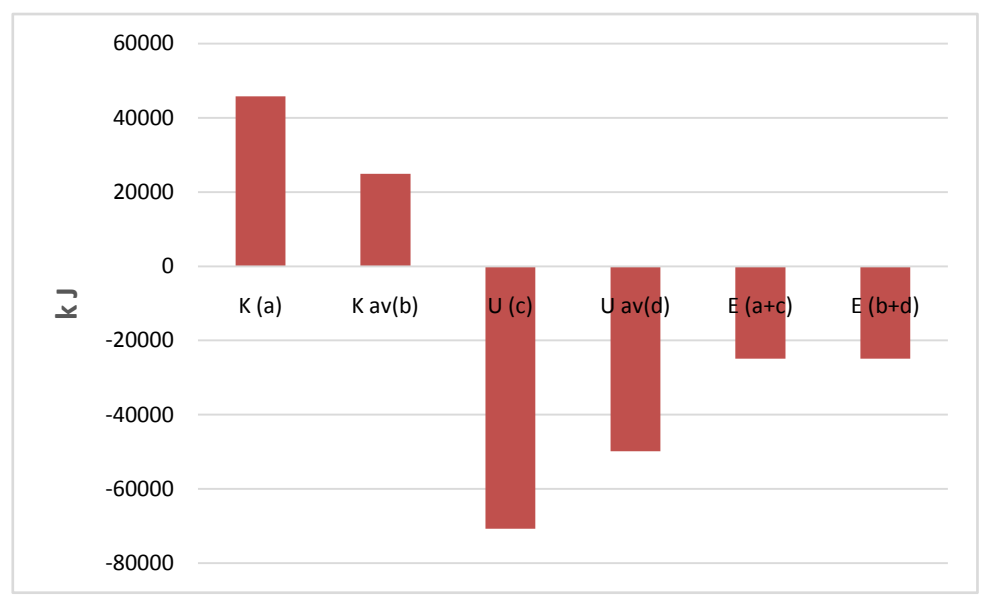

Figure 8. Gravitational energy.

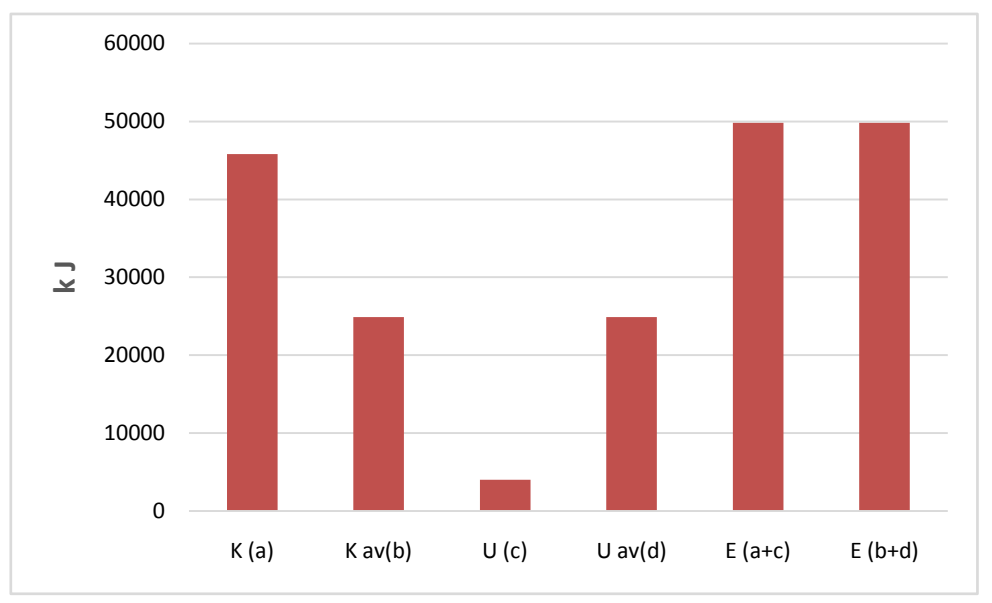

Figure 9. Elastic energy.

The computed kinetic energy shown in Figure 10 was obtained using Equation (19) and compared with that one obtained using the velocity from Vis-viva equation. Its average value was obtained using Equation (20).

Figure 11 shows the computed kinetic power obtained using Equation (33) and compared with the time derivative of the original one, obtained using the Vis-viva equation.

Figure 12 shows the gravitational potential energy variation (Equation (21)) using Keppler's equations and Hook's.

\section{A Proposition for the Starting Point for a Practical Synthesis of the Quantities Related to Orbital Motions}

As we have seen that closed gravitational trajectories on a plane can be interpreted as a composition of periodic motions on each axis of a system of orthogonal coordinates, generating the known Lissajous figures, we can extend this concept for motions on three symmetrically displaced axes, named phase, $a b$ and $c$ axes, given respectively by the unity vectors $a^{0}, a$ and $a^{2}$ where 


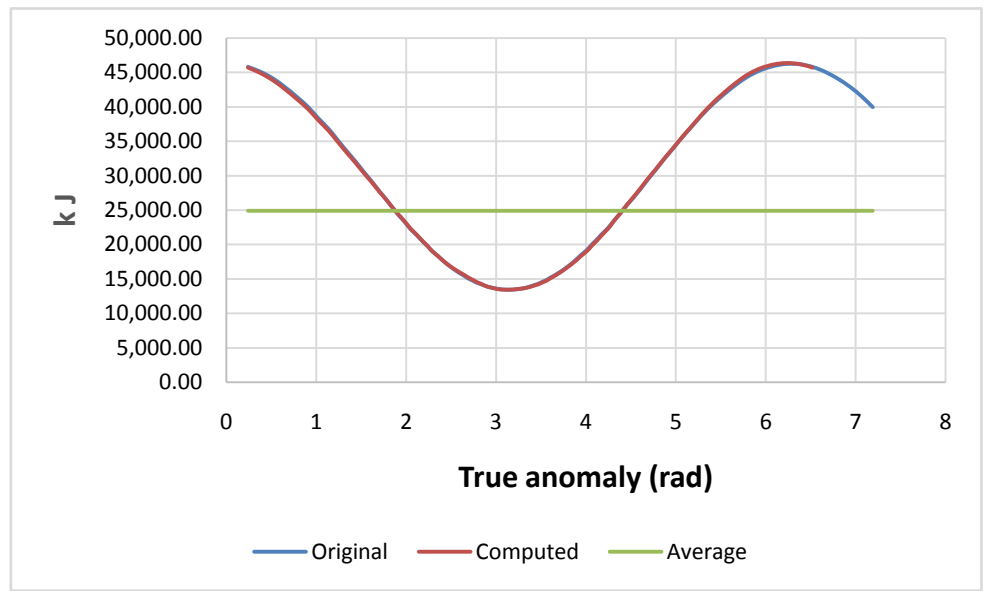

Figure 10. Kinetic energy variation.

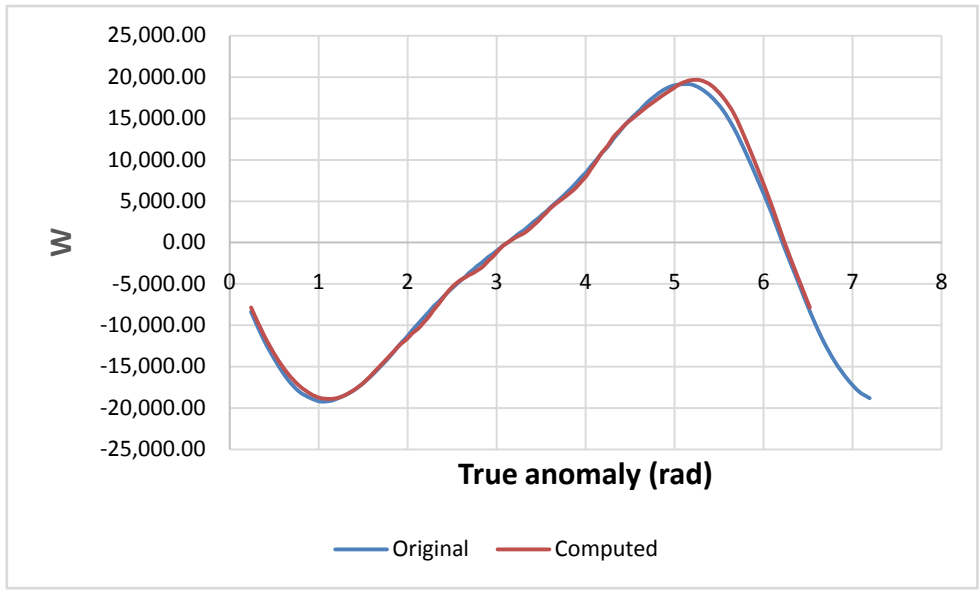

Figure 11. Kinetic power variation.

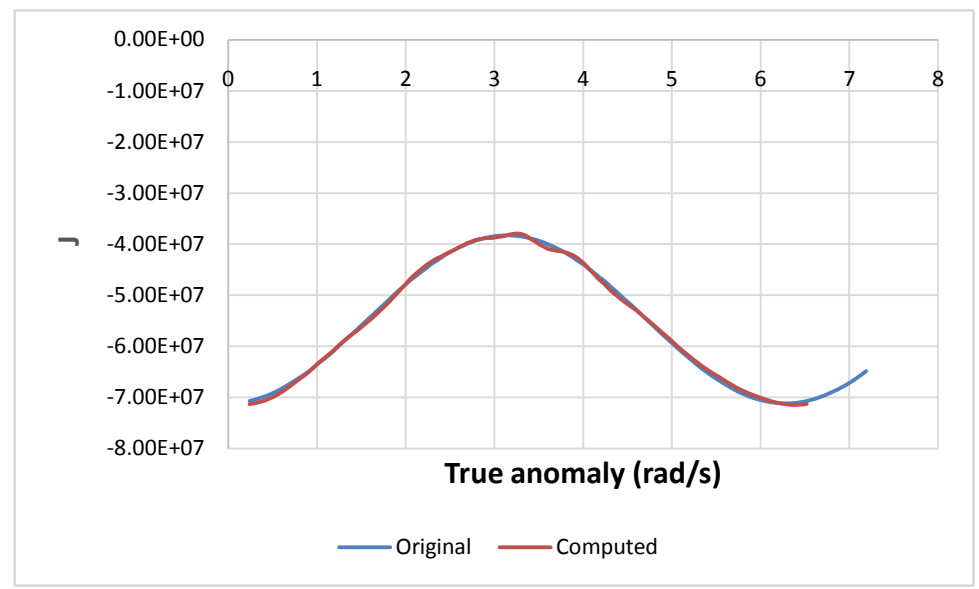

Figure 12. Gravitational potential energy variation.

$a=\mathrm{e}^{i \frac{2 \pi}{3}}$, on a plane (more axes are possible too), using the inverse transformation of Clarke's transformation 


$$
\tilde{\rho}=\frac{2}{3}\left(\rho_{a}+a \rho_{b}+a^{2} \rho_{c}\right)=\rho_{\alpha}+i \rho_{\beta}
$$

where $\rho_{a}, \rho_{b}$ and $\rho_{c}$ are periodical oscillations respectively on each axis, called phase quantities.

To do that inverse operation, knowing the position vector $\tilde{\rho}$, its projection on axes $a b$ and $c$ gives, respectively, the scalar quantities $\rho_{a}, \rho_{b}$ and $\rho_{c}$ [6].

Looking the position vector in this way, they can be called instantaneous space vectors (ISVs), by analogy with electrical polyphase circuits, where they can represent voltages, currents and fluxes (the correspondent electrical variables are respectively charge for position in the mechanical system $\rho \leftrightarrow q$, current for velocity $v \leftrightarrow i$ and voltage for force $f \leftrightarrow v)$.

The property of ISVs carrying positive, negative and zero sequence components were studied by Akagy, Ferreiro, Willems, Emanuel and the author [3] [4] [5] [6] [8] [9]. But, in fact, that property, for only the cases of positive and negative sequences, is evidenced in the terns of the Fourier series. Fortescue deals with those components on phase analyses of unbalanced three-phase electrical circuits, Clarke with both the three-phase and the equivalent two-phase circuit obtained after Clarke transformation, and the complex Fourier series approach deals only with the equivalent two-phase circuit, without mentioning the possibly relations with $\mathrm{n}$-phase oscillating systems.

Thus, having stablished those relations between three-phase systems and the equivalent two-phase systems, synthesis of ISVs may be carried out using power electronics techniques and electromechanical devices. In this way, many alternatives can be proposed for future research; more precisely, acting with conventional propulsion systems or hybrid electromechanical systems having in mind that each harmonic variable of positive and/or negative sequence can be controlled individually or in harmonic ranges.

Using equivalent three-phase mechanical or electrical systems (motions in three symmetrical directions), the kinetic and potential stored energies are respectively given as ([6], Appendix)

$$
\begin{gathered}
K=\frac{3}{4} m V^{2} \Leftrightarrow E_{L}=\frac{3}{4} L I^{2}, \\
U_{\text {Hook }}=\frac{3}{4} F_{G} \rho \Leftrightarrow E_{U}=\frac{3}{4} C V^{2},
\end{gathered}
$$

where $E_{L}, L$ and $I$ are respectively magnetic energy, inductance and current and $E_{U}, C$ and $V$, respectively, potential energy, capacitance and voltage.

Those energy equations show that the oscillating variables on each direction (phase on electrical systems) of a three-axis mechanical motion have their magnitudes decreased, which means material savings and more energy storage capacity.

More precisely, speaking of a spacecraft, each harmonic motion following an elliptical trajectory, composed of positive and negative sequence motions, may be canceled by an opposing motion produced, for example, by the composition 
of the motions of two internal rotating bodies (each rotation in the conventional manner to produce artificial gravity and attached to the center of the spacecraft by means of an elastic spring or an equivalent solenoid electromagnetic damper), which rotates in opposite directions to cancel, respectively, the corresponding positive and negative harmonic motions of the spacecraft or, for a more practical solution, each harmonic trajectory with its correspondent variables may be obtained using two orthogonal coordinated internal harmonic motions, or three symmetrical coordinated internal harmonic motions, produced for example by two (or three) magnetic pistons moving inside a cylindrical electrically controlled solenoid (a kind of active linear solenoidal dampers) or linear electromagnetic motors. For these cases, Equations (35) and (36) may be considered.

This study is in the field of mechanical vibrations and the resonance phenomena applied to the motions of two bodies, joined by an elastic device, moving in a gravitational field. All the above mentioned process is a tuning process between the chosen natural frequency motion of the spacecraft and the artificial vibrating system.

The complex geometry of the total motion as well as of each harmonic component must be carefully considered.

This method must be applied first to harmonic motions of high frequency, since the amount of energy is smaller than those of low frequencies, as well as their amplitudes and centripetal forces; which implies easier practical implementation, to cancel the gravitational components of a range of harmonic motions. In this way, the spacecraft's original elliptical motion takes another elliptical motion with less harmonic contents.

The greater the eccentricity of the orbit, the greater the amount of harmonic energy that can be dealt with using practical applications, because, as we have seen, harmonic frequencies increase, whose correspondent trajectories are smaller; what decreases the dimensions of the electromechanical systems.

Additionally, the results shown in this work point to the possibility that a free fall of an object close to the earth surface, because, due the tangential velocity of the earth location, they follow an elliptical trajectory with eccentricity approaching to one (before hitting the ground). In this case there must be harmonics of high orders, which may be tuned with electromechanical devices. All these considerations are all hypothetical and must be proven practically.

For applications near the earth surface there are anchorages on conventional anchor foundations.

\section{Conclusions}

A numerical simulation of an elliptical orbital motion was implemented in this work, using Fourier analysis and synthesis of the related mechanical quantities, as well as, an energy and power study.

The great differential of the used methodology was the complementary use of Fortescue and Clark's methods of analysis. 
The results confirmed a new concept for gravitational forces, which stablishes that “Newton's Universal Law for gravitational forces on orbital motions can be considered as a sum of harmonic forces of Hook's elastic type, which is a weighted series of harmonic position vectors".

The two summations on the right-hand side of Equation (4) show that the elliptical motion of a satellite around a central body is composed of $\mathrm{N}$ harmonic elliptical motions, each one produced by the composition of positive sequence and the negative sequence circular trajectories.

The method here employed is also an alternative way to calculate the orbital velocity magnitude and angle.

The numerical results shown in the tables, as well as in the figures, validated the method with good precision through the comparison of all the quantities produced from Kepler and Newton's law and those produced in terms of harmonic vectors despite of limitations of the computational means employed. In this way, it was numerically verified that Newtons inverse square law for gravitational forces, on orbital motions, can be thought as a series of harmonic forces of Hooks elastic types or, in other words, as a weighted series of harmonic position vectors.

The following properties were verified for this type of motion:

The number of harmonics depends on the ellipses eccentricity.

The initial harmonic phase angles for the displacement vectors are multiple of the mean anomaly $M$ :

$$
\varphi_{\rho h 0}^{+}=\varphi_{\rho h 0}^{-}=h M
$$

The Vis-viva equation as well as Newton's inverse square law equation can be, alternatively, replaced by Equations (10) and (13), respectively, for obtaining the correspondent quantities. The Vis-viva equation gives only the magnitude of the velocity vector, but Equations (10) and (11) give both its magnitude and its angle.

It was observed, with the adopted approximation [10], that the negative sequence vectors magnitudes, as well as the harmonic components of the Fourier series, increase with the ellipses eccentricity for simulations with eccentricity varying between zero and 0.3 . Thus, it is expected that this tendency is true for eccentricity values tending to 1 (radial trajectory), which is almost a right line trajectory and the number of harmonics tends to infinity, and the positive and negative sequence vector tend to equality. This case can be thought as a free fall of a body.

If those hypotheses are confirmed, this methodology can be extended to non-periodical motions, using Fourier transform and the harmonic spectrum is almost symmetrical regarding to the axis passing through zero frequency. Also, this methodology can be extended to the cinematics and dynamics of any planar periodical, and, if the above hypothesis is confirmed, non-periodical motions. Moreover, an analogy between three-phase electrical circuits and gravitational motions was evidenced. Indeed, the theory here presented is implicit on the 
study of power and energy stored on capacitor banks. Thus, the same considerations can be done for electrical systems, concerning those quantities: the stored energy on a capacitor bank can be given as the product of voltage and electrical charge, shifting in this way the reference point from the coordinate system origin to infinity.

Those conclusions bring forth the idea that gravitational forces can be obtained by, for example, electromechanical devices and, in this way, new techniques for satellite navigations can be developed, as well as new forms of gravitational energy extractions.

\section{References}

[1] Fortescue, C.L. (1918) Method of Symmetrical Coordinates Applied to the Solution of Polyphase Networks. AIEE, 37, 1027-1140.

[2] Clarke, E. (1943) Circuit Analysis of AC Power Systems. Wiley, Hoboken, Chapter 10,308 .

[3] Akagi, H., Kanazawa, Y. and Nabae, A. (1983) Generalized Theory of Instantaneous Reactive Power in Three-Phase Circuits. IPEC-83, Tokyo, 1375-1386.

[4] Ferreiro, A. and Superti, G. (1991) A New Approach to the Definitions of Power Components in Three-Phase Systems under Nonsinusoidal Conditions. IEEE Transactions on Instrumentation and Measurement, 40, 568-577. https://doi.org/10.1109/19.87021

[5] Willems, J.L. (1992) A New Interpretation of Akagi-Nabae Power Components. IEEE Transactions on Instrumentation and Measurement, 41, 523-527. https://doi.org/10.1109/19.155919

[6] Milanez, D.L. and Emanuel, A.E. (2003) The Instantaneous-Space-Phasor a Powerful Diagnosis Tool. IEEE Transactions on Instrumentation and Measurement, 52 143-148.

[7] Emanuel, A.E. (2010) Power Definitions and the Physical Mechanism of Power Flow. IEEE Press and Wiley, 264. https://doi.org/10.1002/9780470667149

[8] Gossick, B.R. (1967) Hamilton's Principle and Physical Systems. Academic Press, New York and London, 247.

[9] Akagi, H. and Nabae, A. (1983) The p-q Theory in Three-Phase Systems under Non-Sinusoidal Conditions. European Transactions on Electrical Power, 3, 27-31. https://doi.org/10.1002/etep.4450030106

[10] Randal, D.P. (1991) True Anomaly Approximation for Elliptical Orbits. Journal of Guidance, Control, and Dynamics, 14, 1069-1070. https://doi.org/10.2514/3.20754 


\section{Appendix A}

The harmonic component of position vector

$$
\tilde{\rho}_{h}=\tilde{\rho}_{h}^{+}+\tilde{\rho}_{h}^{-},
$$

where

$$
\begin{gathered}
\tilde{\rho}_{h}^{+}=\rho_{h}^{+} \mathrm{e}^{i\left(\varphi_{\rho h}^{+}\right)}=\rho_{h}^{+} \mathrm{e}^{i\left(h \omega_{1} t+\varphi_{\rho h 0}^{+}\right)}=\dot{\rho}_{h}^{+} \mathrm{e}^{i\left(h \omega_{1} t\right)}, \\
\tilde{\rho}_{h}^{-}=\rho_{h}^{-} \mathrm{e}^{-i\left(\varphi_{\rho h}^{-}\right)}=\rho_{h}^{-} \mathrm{e}^{-i\left(h \omega_{1} t-\varphi_{\rho h 0}^{-}\right)}=\dot{\rho}_{h}^{-} \mathrm{e}^{-i\left(h \omega_{1} t\right)},
\end{gathered}
$$

expressed on Cartesian coordinates,

$$
\begin{aligned}
\tilde{\rho}_{h}= & \rho_{h}^{+}\left[\cos \left(h \omega_{1} t+\varphi_{\rho h 0}^{+}\right)+i \sin \left(h \omega_{1} t+\varphi_{\rho h 0}^{+}\right)\right] \\
& +\rho_{h}^{-}\left[\cos \left(h \omega_{1} t-\varphi_{\rho h 0}^{-}\right)-i \sin \left(h \omega_{1} t-\varphi_{\rho h 0}^{-}\right)\right]
\end{aligned}
$$

at the perihelion position where $\varphi_{\rho h 0}^{+}=\varphi_{\rho h 0}^{-}=0$, results that

$$
\tilde{\rho}_{h}=\left(\rho_{h}^{+}+\rho_{h}^{-}\right) \cos \left(h \omega_{1} t\right)+i\left(\rho_{h}^{+}-\rho_{h}^{-}\right) \sin \left(h \omega_{1} t\right) .
$$

Now, considering that $\rho_{h}^{+}+\rho_{h}^{-}=a$, the semi-major ellipses' axis, and $\rho_{h}^{+}-\rho_{h}^{-}=b$, the semi-minor axis, the ellipses' condition,

$$
\frac{a^{2} \cos ^{2}\left(h \omega_{1} t\right)}{a^{2}}+\frac{b^{2} \sin ^{2}\left(h \omega_{1} t\right)}{b^{2}}=1
$$

is fulfilled.

\section{Appendix B}

The following are the expressions of the positive and negative sequence harmonic position vectors:

$$
\begin{gathered}
\tilde{\rho}_{h}^{+}=\rho_{h}^{+} \mathrm{e}^{i\left(\varphi_{\rho h}^{+}\right)}=\rho_{h}^{+} \mathrm{e}^{i\left(h \omega_{1} t+\varphi_{\rho h 0}^{+}\right)}=\dot{\rho}_{h}^{+} \mathrm{e}^{i\left(h \omega_{1} t\right)}, \\
\tilde{\rho}_{h}^{-}=\rho_{h}^{-} \mathrm{e}^{-i\left(\varphi_{\rho h}^{-}\right)}=\rho_{h}^{-} \mathrm{e}^{-i\left(h \omega_{1} t-\varphi_{\rho h 0}^{-}\right)}=\dot{\rho}_{h}^{-} \mathrm{e}^{-i\left(h \omega_{1} t\right)},
\end{gathered}
$$

their first derivatives

$$
\begin{gathered}
\frac{\mathrm{d} \tilde{\rho}_{h}^{+}}{\mathrm{d} t}=i h \omega_{1} \dot{\rho}_{h}^{+} \mathrm{e}^{i\left(h \omega_{1} t\right)}=i h \omega_{1} \tilde{\rho}_{h}^{+}, \\
\frac{\mathrm{d} \tilde{\rho}_{h}^{-}}{\mathrm{d} t}=-i h \omega_{1} \dot{\rho}_{h}^{-} \mathrm{e}^{-i\left(h \omega_{1} t\right)}=-i h \omega_{1} \tilde{\rho}_{h}^{-}
\end{gathered}
$$

and second derivatives

$$
\begin{aligned}
& \frac{\mathrm{d}\left(\frac{\mathrm{d} \tilde{\rho}_{h}^{+}}{\mathrm{d} t}\right)}{\mathrm{d} t}=i^{2} h^{2} \omega_{1}^{2} \tilde{\rho}_{h 1}^{+}, \\
& \frac{\mathrm{d}\left(\frac{\mathrm{d} \tilde{\rho}_{h}^{-}}{\mathrm{d} t}\right)}{\mathrm{d} t}=i^{2} h^{2} \omega_{1}^{2} \tilde{\rho}_{h 1}^{-} .
\end{aligned}
$$




\section{Appendix C}

The constant gravitational energy is the sum of the instantaneous kinetic and potential energies, and equal to the sum of their average values:

$$
E_{G}=K+U_{G}=\bar{K}+\overline{U_{G}} .
$$

In turn, the average value of the kinetic energy is half the average potential energy value, with opposite sign:

$$
\bar{K}=-\frac{1}{2} \overline{U_{G}} .
$$

Thus,

$$
\bar{K}=-E_{G}
$$

For the constant elastic energy,

$$
E_{\text {Hook }}=k+U_{\text {Hook }}=\bar{K}+\overline{U_{\text {Hook }}} \text {. }
$$

But, in this case, the average value of the kinetic energy is equal to the average potential energy value,

$$
\bar{K}=\overline{U_{\text {Hook }}} \text {. }
$$

Therefore,

$$
\bar{K}=\frac{1}{2} E_{\text {Hook }}
$$

As the kinetic energy has the same value on any reference system, comparing the kinetic energy of both systems, imply that

$$
E_{G}=-\frac{1}{2} E_{\text {Hook }} \text {. }
$$

Q.E.D.

\section{Appendix D}

In this Appendix, we are going to see how is the power contribution of every harmonic motion (positive and negative sequences harmonic motions) to the total power given as the derivative of kinetic energy given by Equation (14); separating them as harmonic powers related to forces and velocities of same harmonic order, and harmonic powers related to forces and velocities of different harmonic orders.

The instantaneous complex power (ICP) concept is presented in [4] [5] [6] [8] [9]. ICP is a time-varying complex quantity given by

$$
\begin{aligned}
\tilde{S} & =\tilde{F} \tilde{V}^{*}=\left(\tilde{F}^{+}+\tilde{F}^{-}\right)\left(\tilde{V}^{+}+\tilde{V}^{-}\right)^{*} \\
& =\tilde{F}^{+} \tilde{V}^{+}+\tilde{F}^{+} \tilde{V}^{-}+\tilde{F}^{-} \tilde{V}^{+}+\tilde{F}^{-} \tilde{V}^{-}
\end{aligned}
$$

here

$$
\tilde{F}^{+}=m \frac{\mathrm{d} \tilde{V}^{+}}{\mathrm{d} t} \text { and } \tilde{F}^{-}=m \frac{\mathrm{d} \tilde{V}^{-}}{\mathrm{d} t}
$$




\section{D1. Harmonic Powers Due to Forces and Velocities of Same Harmonic Order}

For each harmonic pair of force and velocity, the ICP is

$$
\begin{aligned}
\tilde{S}_{h} & =\tilde{F}_{h} \tilde{V}_{h}^{*}=\left(\tilde{F}_{h}^{+}+\tilde{F}_{h}^{-}\right)\left(\tilde{V}_{h}^{+}+\tilde{V}_{h}^{-}\right)^{*} \\
& =\dot{F}_{h}^{+}\left(\dot{V}_{h}^{+}\right)^{*}+\dot{F}_{h}^{-}\left(\dot{V}_{h}^{-}\right)^{*}+\tilde{F}_{h}^{+}\left(\dot{V}_{h}^{-}\right)^{*}+\tilde{F}_{h}^{-}\left(\dot{V}_{h}^{+}\right)^{*} \\
& =S_{h}^{+}+S_{h}^{-}+S_{h}^{ \pm}+S_{h}^{\mp}
\end{aligned}
$$

The first two terms on the right-hand side of this expression, $S_{h}^{+}$and $S_{h}^{-}$, are constant and are called complex powers, and the other two, $\tilde{S}_{h}^{ \pm}$and $\tilde{S}_{h}^{+}$ are oscillating powers, and are called ICPs.

The real part of $S_{h}^{+}$and $S_{h}^{-}, P_{h}^{+}$and $P_{h}^{-}$, respectively, are the positive and the negative sequence real powers and are nil in the case of non-dissipative motions (for example: no atmosphere resistance):

$$
\begin{aligned}
& P_{h}^{+}=\operatorname{Real}\left(S_{h}^{+}\right)=0 \\
& P_{h}^{-}=\operatorname{Real}\left(S_{h}^{-}\right)=0
\end{aligned}
$$

and the imaginary part, $Q_{h}^{+}$and $Q_{h}^{-}$, respectively, are the positive and the negative sequence imaginary powers and they are constant:

$$
\begin{gathered}
Q_{h}^{+}=\operatorname{Imag}\left(S_{h}^{+}\right)=h \omega m\left(V_{h}^{+}\right)^{2} \\
Q_{h}^{-}=\operatorname{Imag}\left(S_{h}^{-}\right)=-h \omega m\left(V_{h}^{-}\right)^{2}
\end{gathered}
$$

Real powers are related to force vectors in phase with velocity vectors, and imaginary powers are related to those vectors in quadrature.

As we are interpreting the orbital motion as two coordinated motions, on two orthogonal axes on a plane, the physical meaning of these imaginary powers is that they are the pick values of the rate of kinetic energy exchanged between the two axes, which are sinusoidal quantities, respectively on the equivalent positive and negative sequence motions (the same interpretation is used to define reactive powers on electrical circuit analysis).

Note that they are related to the harmonic sequence kinetic energies in this way:

$$
\begin{gathered}
K_{h}^{+}=\frac{1}{2} m\left(V_{h}^{+}\right)^{2}=\frac{Q_{h}^{+}}{2 h \omega} \\
K_{h}^{-}=\frac{1}{2} m\left(V_{h}^{-}\right)^{2}=-\frac{Q_{h}^{-}}{2 h \omega}
\end{gathered}
$$

where $K_{h}^{+}$and $K_{h}^{-}$are, respectively, the kinetic energy associated to the harmonic positive and negative sequence motions. They are constant quantities.

The sum of these two harmonic kinetic energies given by

$$
K_{h}^{ \pm}=K_{h}^{+}+K_{h}^{-}
$$

is the average kinetic energy during a cycle, related to this harmonic motion (see 
equation 15):

$$
K_{\text {havg }}=K_{h}^{ \pm}=\overline{\left(\frac{1}{2} m V_{h}^{2}\right)}
$$

The amount

$$
K_{a v}=\sum_{h=1}^{N}\left(K_{h}^{+}+K_{h}^{-}\right)
$$

is the average kinetic energy of the total motion during one cycle.

Considering now the ICP terms $\tilde{S}_{h}^{ \pm}$and $\tilde{S}_{h}^{\mp}$ of the expression for $\tilde{S}_{h}$, we have that, as in the cases of the above mentioned real and imaginary powers, their real $\tilde{Q}_{P h}^{ \pm}, \tilde{Q}_{P h}^{\mp}$ and imaginary $\tilde{Q}_{Q h}^{ \pm}, \tilde{Q}_{Q h}^{\mp}$ parts are also results of force and velocity components, respectively in phase and in quadrature. But, here, these components are not constant during a cycle of the motion, i.e., they vary with frequency $2 h \omega$, and their average values are nil (in an analogous electrical circuit, due the fact that the elements of the analyzed system are storage energy, or reactive, elements, even been powers produced by voltages and in-phase current vectors, these powers are named instantaneous reactive powers, or nonactive powers ([7], p. 156). The term inactive is due to the fact that the power flow is a system internal power flow. Thus, the same denominations are adopted here. It can be verified for these harmonic instantaneous reactive powers that

$$
\tilde{Q}_{P h}^{ \pm}=\tilde{Q}_{P h}^{\mp}
$$

and

$$
\tilde{Q}_{Q h}^{ \pm}+\tilde{Q}_{Q h}^{\mp}=0 .
$$

The physical meaning of this is that there is a power oscillation between the kinetic form and the gravitational form for each harmonic order motion, given by

$$
\tilde{Q}_{P h}=\tilde{Q}_{P h}^{ \pm}+\tilde{Q}_{P h}^{\mp}=2 \tilde{Q}_{P h}^{ \pm} .
$$

Thus, the subtotal power oscillation, which is due to all harmonic pairs is

$$
\tilde{Q}_{\sum P h}=\tilde{Q}_{\Sigma P h}^{+-}+\tilde{Q}_{\Sigma P h}^{-+}=\sum \operatorname{Real}\left[\tilde{F}_{h}^{+}\left(\tilde{V}_{h}^{-}\right)^{*}+\tilde{F}_{h}^{-}\left(\tilde{V}_{h}^{+}\right)^{*}\right] .
$$

\section{D2. Harmonic Powers Due to Forces and Velocities of Different Harmonic Orders}

There are also oscillating powers between the potential field and the moving mass due to forces and velocities of different harmonic orders. Let us represent them by $\tilde{S}_{i j}^{ \pm}$and $\tilde{S}_{i j}^{\mp}$, given respectively by

$$
\tilde{S}_{i j}^{ \pm}=\tilde{F}_{i}^{+}\left(\tilde{V}_{I}^{+}\right)^{*}+\tilde{F}_{i}^{+}\left(\tilde{V}_{I}^{-}\right)^{*}
$$

and

$$
\tilde{S}_{i j}^{\mp}=\tilde{F}_{i}^{-}\left(\tilde{V}_{I}^{+}\right)^{*}+\tilde{F}_{i}^{-}\left(\tilde{V}_{I}^{-}\right)^{*}
$$


The real part of these powers

$$
\tilde{Q}_{P i j}^{ \pm}=\operatorname{Real}\left(\tilde{S}_{i j}^{ \pm}\right)
$$

and

$$
\tilde{Q}_{Q i j}^{\mp}=\operatorname{Real}\left(\tilde{S}_{i j}^{\mp}\right)
$$

are the rate of energy exchanged between the moving mass of the harmonic motion of order $i$ and the potential field of harmonic motion of order $j$.

Thus, the subtotal power oscillation due to all harmonic of different orders is

$$
\begin{aligned}
\tilde{Q}_{\sum P i j} & =\tilde{Q}_{\sum P i j}^{ \pm}+\tilde{Q}_{\sum P i j}^{\mp} \\
& =\sum_{i=1}^{N} \sum_{\substack{j=1 \\
j \neq i}}^{N} \operatorname{Real}\left[\tilde{F}_{i}^{+}\left(\tilde{V}_{j}^{+}\right)^{*}+\tilde{F}_{i}^{+}\left(\tilde{V}_{j}^{-}\right)^{*}+\tilde{F}_{i}^{-}\left(\tilde{V}_{j}^{+}\right)^{*}+\tilde{F}_{i}^{-}\left(\tilde{V}_{j}^{-}\right)^{*}\right]
\end{aligned}
$$

and the total power oscillation is the summation of these two subtotal powers

$$
\tilde{Q}_{\sum P}=\tilde{Q}_{\sum P h}+\tilde{Q}_{\sum P i j}=\tilde{Q}_{\sum P h}^{ \pm}+\tilde{Q}_{\sum P h}^{\mp}+\tilde{Q}_{\sum P i j}^{ \pm}+\tilde{Q}_{\sum P i j}^{\mp}
$$

NOTE ${ }^{*}$ : the zero-sequence vector has another meaning in electrical circuit analysis. 